\title{
openheart Mechanisms of tissue uptake and retention of paclitaxel-coated balloons: impact on neointimal proliferation and healing
}

\author{
Juan F Granada, ${ }^{1}$ Mark Stenoien, ${ }^{2}$ Piotr P Buszman, ${ }^{1}$ Armando Tellez, ${ }^{1}$ \\ Dan Langanki, ${ }^{2}$ Greg L Kaluza, ${ }^{1}$ Martin B Leon, ${ }^{3}$ William Gray, ${ }^{3}$ Michael R Jaff, ${ }^{4}$ \\ Robert S Schwartz ${ }^{5}$
}

To cite: Granada JF, Stenoien M, Buszman PP, et al. Mechanisms of tissue uptake and retention of paclitaxel-coated balloons: impact on neointimal proliferation and healing. Open Heart 2014;1:e000117 doi:10.1136/openhrt-2014000117

Received 3 March 2014 Revised 5 May 2014 Accepted 28 May 2014

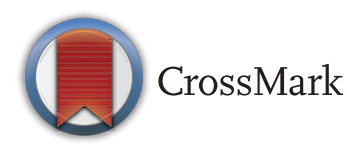

${ }^{1}$ Skirball Center for Cardiovascular Research, Cardiovascular Research Foundation, Orangeburg, New York, USA ${ }^{2}$ MEDRAD Interventional, Indianola, Pennsylvania, USA ${ }^{3}$ Columbia University Medical Center, Center for Interventional Vascular Therapy, New York Presbyterian Hospital, New York, New York, USA ${ }^{4}$ Harvard Medical School, Massachusetts General Hospital, Boston,

Massachusetts, USA

${ }^{5}$ Minneapolis Heart Institute and Foundation, Abbott Northwestern Hospital, Minneapolis, Minnesota, USA

Correspondence to Dr Juan F Granada; jgranada@crf.org

\section{ABSTRACT}

Background: The efficacy of paclitaxel-coated balloons (PCB) for restenosis prevention has been demonstrated in humans. However, the mechanism of action for sustained drug retention and biological efficacy following single-time drug delivery is still unknown.

Methods and results: The pharmacokinetic profile and differences in drug concentration (vessel surface vs arterial wall) of two different paclitaxel coating formulations $\left(3 \mu \mathrm{g} / \mathrm{mm}^{2}\right)$ displaying opposite solubility characteristics $(C C=$ crystalline vs $A C=$ amorphous) were tested in vivo and compared with paclitaxel-eluting stents (PES). Also, the biological effect of both PCB formulations on vascular healing was tested in the porcine coronary injury model. One hour following balloon inflation, both formulations achieved similar arterial paclitaxel levels (CC $=310$ vs $A C=245 \mathrm{ng} / \mathrm{mg}$; $\mathrm{p}=\mathrm{NS}$ ). At $24 \mathrm{~h}$, the $\mathrm{CC}$ maintained similar tissue concentrations, whereas the AC tissue levels declined by $99 \%(p<0.01)$. At this time point, arterial levels were 20 -fold (CC) and 5 -fold (AC) times higher compared to the PES group $(p<0.05)$. At 28 days, arterial levels retained were $9.2 \%(C C)$ and $0.04 \%(A C, p<0.01)$ of the baseline levels. Paclitaxel concentration on the vessel surface was higher in the $\mathrm{CC}$ at 1 ( $C \mathrm{C}=36.7 \%$ vs $A C=13.1 \%, p<0.05)$ and 7 days $(C C=38.4 \%$ vs $A C=11 \%$, $\mathrm{p}<0.05)$. In addition, the $\mathrm{CC}$ induced higher levels of neointimal inhibition, fibrin deposition and delayed healing compared with the AC group.

Conclusions: The presence of paclitaxel deposits on the vessel surface driving diffusion into the arterial tissue in a time-dependent fashion supports the mechanism of action of PCB. This specific pharmacokinetic behaviour influences the patterns of neointimal formation and healing.

\section{INTRODUCTION}

Paclitaxel-coated balloons (PCB) have emerged as a viable therapeutic alternative for the interventional treatment of vascular disease. ${ }^{1-5}$ With the use of this technology, the

\section{KEY MESSAGES}

What is already known about this subject?

- The efficacy of paclitaxel-coated balloons (PCB) for restenosis prevention has been demonstrated in humans. However, the mechanism of action for sustained drug retention and biological efficacy following single-time drug delivery is still unknown.

What does this study add?

- Our data suggest that the observed tissue halflife of paclitaxel delivered by PCB technologies is rate limiting and relates to the slow dissolution of paclitaxel deposits from the vessel surface into arterial tissue in a time-dependent fashion.

How might this impact on clinical practice?

- This experimental study provides better understanding of the processes of paclitaxel local delivery which may implicate in the refinement of currently available and development of new treatment technologies and options for atherosclerotic vascular disease.

short-term transfer and long-term retention of paclitaxel to the arterial wall is feasible,${ }^{16}$ thus potentially reducing the untoward effects of the prolonged drug release associated with polymer-based stent technologies ${ }^{7-9}$ including delayed vessel healing, potentially resulting in stent thrombosis. Experimental data have shown that some paclitaxel formulations are rapidly transferred to the vessel wall following balloon dilation. ${ }^{10}{ }^{11}$ However, it has been suggested that long-term tissue retention is affected by the drug morphology and resulting solubility attained during the coating process. ${ }^{12}$

First-generation PCB technologies displayed a high level of crystallinity on the coating surface and were associated with inconsistent drug load and high particulate 
shedding during balloon inflation. New generations of PCB coatings are under development, displaying more consistent, uniform and less crystalline (CC) coating characteristics. Although these technological changes may potentially affect clinical outcomes, the resulting biological effects on paclitaxel uptake and vessel healing are still unknown.

At the present time, a potential mechanism of action explaining long-term drug retention yielding sustained biological efficacy following single-time drug delivery has not been described. In this study, we compared the impact of two different coating types on tissue transfer, retention and biological efficacy and propose a mechanism of action explaining the durability of the biological effect following single-time delivery of paclitaxel via balloon delivery.

\section{METHODS}

\section{Device description}

PCB angioplasty catheters were prepared having two distinct drug solid-state morphologies using identical balloon platforms and paclitaxel-iopromide coating concentrations applied at a total drug dose of $3 \mu \mathrm{g} / \mathrm{mm}^{2}$. The solution was precisely metered onto all surfaces of the balloons, including within the folds. As a consequence of the proprietary controlled drying process following balloon coating, the resulting paclitaxel morphologies were either substantially amorphous (AC) or dihydrated CC. The morphological form of the coating was confirmed by surface electron microscopy and Raman spectroscopy (figure 1). The comparison paclitaxel-eluting stent (PES) used in this study was the commercially available balloon-expandable TAXUS Liberté stent system (TS, $3 \times 15 \mathrm{~mm}$, Boston Scientific, Natick, Massachusetts, USA) coated with a durable polymeric coating formulation containing paclitaxel at a concentration of $1 \mu \mathrm{g} / \mathrm{mm}^{2}$.

\section{Study schematic}

The study scheme is presented in figure 2. In the first paclitaxel tissue transfer study, abdominal aortas of 44 white rabbits were enrolled. In the following vascular response experiment, 12 domestic swine (DS) were included. The description of each study section is provided below.

\section{Pharmacokinetic studies}

All animals received standard care outlined in accordance with the act of animal welfare and the 'Principles of Care of Laboratory Animals'. ${ }^{13}$ Abdominal aortas of 44 New Zealand white rabbits, either gender, $3.3-4.5 \mathrm{~kg}$, were treated with a single $3.5 \times 20 \mathrm{PCB}$ each at 1.2-1.3:1.0 overstretch ratio for $60 \pm 1 \mathrm{~s}$. An antimicrobial (enrofloxacin, $13.5-17 \mathrm{mg} / \mathrm{kg}$ ), an antispasmodic (Nifedipine, $30 \mathrm{mg}$ ) and aspirin $81 \mathrm{mg}$ were given pre procedure. Induction of anaesthesia was achieved with ketamine/ xylazine $(42-45.5 / 8.75-9 \mathrm{mg} / \mathrm{kg})$ followed by $0-5 \%$ isoflurane propofol $(2-8 \mathrm{mg} / \mathrm{kg})$. Arterial access was obtained using a surgical cut down technique of the

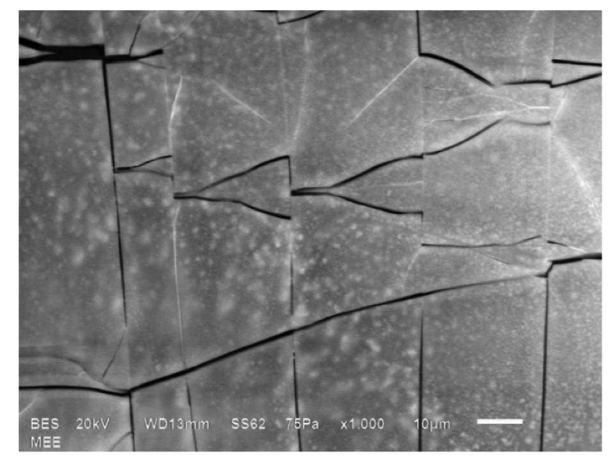

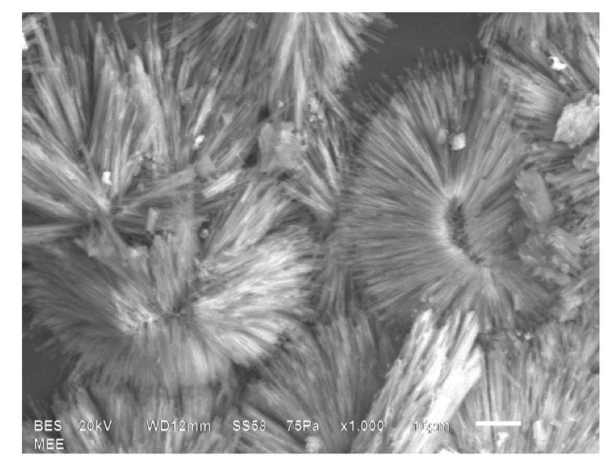

Figure 1 Top panel: surface electron microscopy pictures of crystalline (left) and amorphous (right) coatings based on an identical paclitaxel-iopromide combination. Bottom panel: Raman spectroscopy analysis of both coatings. 
Figure 2 Flow chart describing the study's designs including follow-up times. DS, domestic swine; $A C$, amorphous; $C C$, crystalline; $\mathrm{PCB}$, paclitaxel-coated balloon; POBA, plain balloon angioplasty; OCT, optical coherence tomography; IVUS, intravascular ultrasound.
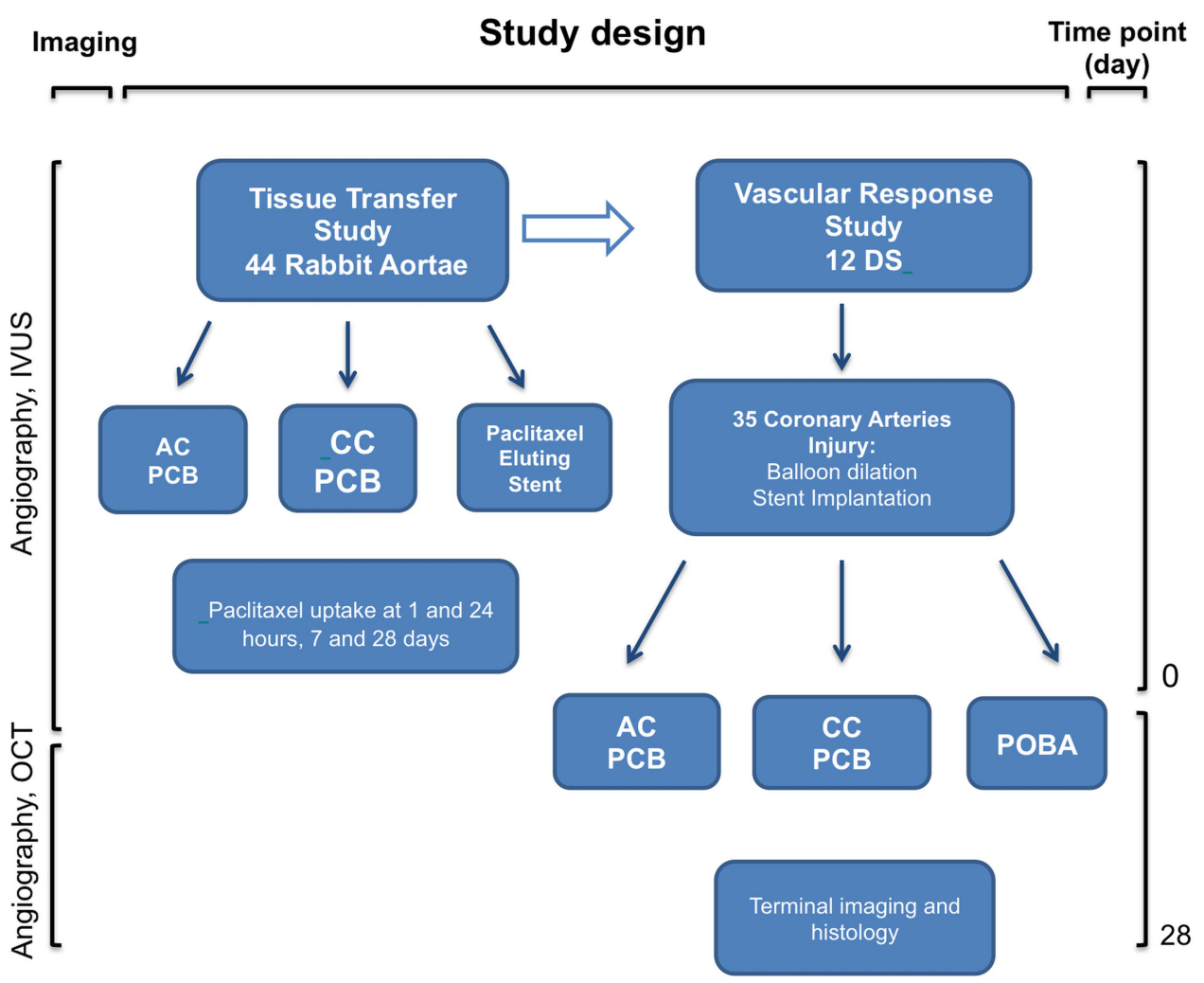

carotid artery. Anticoagulation with heparin was achieved during the procedure to maintain an activated clotting time $\geq 250 \mathrm{~s}$. Treated vessels (four per time point and group) were harvested after $1 \mathrm{~h}, 24 \mathrm{~h}, 7$ and 28 days. Harvested vessels included the $20 \mathrm{~mm}$ treated segment and $10 \mathrm{~mm}$ untreated vessel proximal and distal to the treated segment in order to facilitate handling without damaging the treated segment. Taxus stents $(3 \times 15 \mathrm{~mm})$ were used for comparison in the same model and treated vessels were harvested at $24 \mathrm{~h}, 7$ and 28 days. The total paclitaxel vessel concentration was calculated as a sum of endoluminal and intramural paclitaxel amount divided by total vessel weight prior scraping. The compartmental concentration was calculated as a paclitaxel amount in the endoluminal and intramural compartment divided by the weight of tissue after scraping.

\section{Quantification of paclitaxel surface deposition}

The vessel segment was blotted dry of blood, weighed, carefully incised longitudinally and pinned open, and stents, if present, carefully removed. The vessel surface (endoluminal) paclitaxel was removed together with the entire intimal and endothelial layer from the underlying muscular and adventitial layers of the vessel segment (intramural) by gentle scraping with a blunt plastic pipette. The intima and pipette tip were transferred to a labelled cryovial. The remaining vessel was weighed again and transferred to another labelled cryovial. The cryovials were flash frozen and stored at $-70^{\circ} \mathrm{C}$ prior to analysis for paclitaxel content. Paclitaxel concentrations in the intimal and underlying tissues were determined separately by an independent laboratory using validated liquid chromatography-mass spectrometry (LC-MS)/MS methods. Tissues were sectioned into small pieces and weighed. Samples were then homogenised in phosphate buffered homogenisation solution. The homogenate was labelled with an internal standard, extracted with methyl tert-butyl ether and evaporated to dryness. The reconstituted sample was analysed by reverse-phase highperformance LC with MS/MS detection. The total paclitaxel vessel concentration was calculated as a sum of endoluminal and intramural paclitaxel amount divided by total vessel weight prior scraping. The compartmental concentration was calculated as a paclitaxel amount in the endoluminal and intramural compartment divided by the weight of tissue after scraping. Drug elimination $\left(\mathrm{k}_{\text {elimin }}\right)$ was calculated based on the following formula $\mathrm{k}_{\text {elim }}=\left[\ln \left(\mathrm{C}_{\text {peak }}\right)-\ln \left(\mathrm{C}_{\text {trough }}\right)\right] / \mathrm{t}_{\text {interval }}$. Second, paclitaxel half-life was calculated $\mathrm{t}_{1 / 2}=0.693 / \mathrm{k}_{\text {elim. }}$.

\section{Coronary porcine study}

All animals received standard care outlined in accordance with the act of animal welfare and the 'Principles of Care of Laboratory Animals' ${ }^{13}$ A total of 12 castrated male DS (mean body weight $40.5 \pm 2.4 \mathrm{~kg}$ ) were included and maintained on a standard chow diet until completion of the study. One intramuscular muscarinic anticholinergic dose (glycopyrrolate $0.005-0.02 \mathrm{mg} / \mathrm{kg}$ ) was given pre procedure. Induction of anaesthesia was achieved with a rapid acting general anaesthetic (Tiletamine+Zolazepam, Telazol $2-5 \mathrm{mg} / \mathrm{kg}$ ) followed by 
endotracheal intubation with a continuous inhalation of $1-3 \%$ isoflurane. Arterial access was obtained using a surgical cut down technique of the carotid artery. All animals received $75 \mathrm{mg}$ aspirin and $75 \mathrm{mg}$ clopidogrel 3 days before the procedure and continued until completion of the study. Anticoagulation with heparin was achieved during the procedure (3000-10000 U) to maintain a coagulation time $\geq 250 \mathrm{~s}$. Following baseline angiography, a total of 32 coronary arteries were analysed using intravascular ultrasound to determine the diameters of the coronary target sites. Coronary predilation was performed using a regular angioplasty balloon before stent implantation. Then, balloon-expandable bare metal stents (Orbus X, Bavaria Medizin Technologie GmBH 3 and $3.5 \times 15 \mathrm{~mm}$ ) were deployed at a 1.25:1 overstretch ratio for $30 \pm 3$ s. Finally, stents were dilated with one of the PCB formulations (CC or AC) or a bare balloon was used to postdilate the stents for 30 $\pm 3 \mathrm{~s}$ ensuring at 1:1 PCB ratio. At 28 days follow-up, all animals underwent terminal angiography and optical coherence tomography (OCT) imaging analysis. Quantitative coronary angiography was performed offline by a single operator blinded to the study group utilising QAngio XA Software V.7.1.14.0 (Medis Medical Imaging Systems, Leiden, The Netherlands).

\section{OCT imaging}

OCT images were recorded using the M4 OCT imaging system (ImageWire, LightLab Imaging, Inc, Westford, Massachusetts, USA). Integrated OCT image analysis software developed by LightLab Imaging was used for all measurements. System calibration of the z-offset was performed on each image wire before every single imaging procedure. OCT imaging pullbacks were performed at a rate of $10 \mathrm{~mm} / \mathrm{s}$ using continuous non-occlusive contrast-saline mixture as a flush. All images were acquired at 100 frames/s and stored on the system hard drive for off-line analysis. Morphometric analysis was performed by two operators blinded to the study groups. Each individual pullback analysis was divided into proximal reference vessel, stent segment and distal reference vessel. Instent analysis was divided into 7-8 equal cross sections.

\section{Histology protocol}

Hearts were removed immediately following termination and arteries were flushed with Ringer's lactate for $15 \mathrm{~min}(1 \mathrm{~L})$. After all the blood was washed out, pressure perfusion fixation was performed for 20 min with $10 \%$ neutral buffered formalin. The fixated heart was immersed in a formalin filled container for at least $24 \mathrm{~h}$ to continue further fixation. Coronary arteries were removed from the myocardium and then dehydrated, embedded in plastic and finally sectioned at proximal, mid and distal locations of the stented segment. Each segment was sectioned for staining. One set of sections was stained with $\mathrm{H} \& \mathrm{E}$ and a second set with elastin trichrome stain. Using Spot Advanced V.4.1.1 Software
(Spot Imaging Solutions, Sterling Heights, Michigan, USA), digital vessel images were captured and histomorphometry was performed using NOVA PRIME V.6.70.10 software (Bioquant Image Analysis Corp., Nashville, Tennessee, USA). The Morphometric characteristics that were evaluated were lumen $(\mathrm{L})$ area $\left(\mathrm{mm}^{2}\right)$, internal elastic lamina (IEL) area $\left(\mathrm{mm}^{2}\right)$, external elastic lamina area $\left(\mathrm{mm}^{2}\right)$, medial area $\left(\mathrm{mm}^{2}\right)$, per cent area stenosis and neointimal thickness. Neointimal area was defined as IEL minus L. Percentage area of stenosis was calculated as $[($ IEL-L) $/$ IEL $] \times 100$. A semiqualitative score was used to determine loss of endothelialisation $(0=\geq 90 \%$ of luminal surface covered, $1=75-90 \%$ of luminal surface covered and $2=\leq 75 \%$ of luminal surface covered), inflammation ( $0=$ none, $1=$ mild and $2=$ severe), medial smooth muscle cell (SMC) loss (0=none, $1=1-25 \%$, $2=26-50 \%, 3=51-75 \%, 4=76-100 \%)$ and IEL disruption ( $0=$ none, $1=1-25 \%$ and $2=26-50 \%)$.

\section{Statistical analysis}

Quantitative data were normally expressed as mean value \pm SD. For a skewed distribution, data were expressed as median and IQR. Qualitative data were presented as number and percentage. The Shapiro-Wilk test was used for testing normality. Quantitative data were compared either with the Student $t$ test or one-way analysis of variance (ANOVA) for normal distribution data or the Mann-Whitney U test, otherwise. Holm Sidak post-ANOVA tests were used to test for differences in variables between stent types. When either equal variance test or normality test failed, a Kruskal-Wallis test (with Dunn's method for post hoc group comparison) was conducted; $p$ values of $\leq 0.05$ were considered statistically significant. Areas under the curve (AUCs) were calculated with the trapezoidal rule and compared utilising methodology by Hanley and McNeil. ${ }^{14}$ SigmaStat V.3.11 (Systat Software) and MedCalc V.10.2 (Mariakierke, Belgium) were utilised for the statistical analyses.

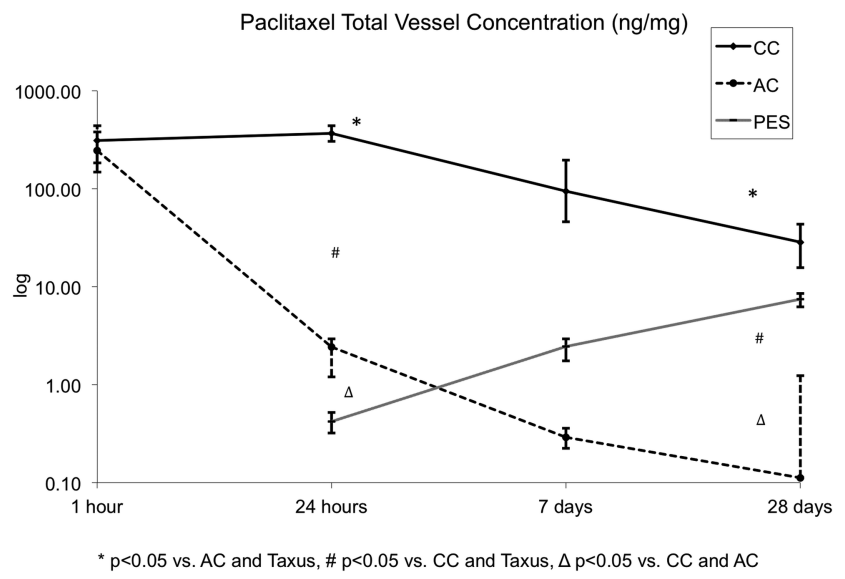

Figure 3 Graphic representation of the 28-day paclitaxel tissue levels of both paclitaxel-coated balloon coatings in comparison with the Taxus stent (PES). AC, amorphous; CC, crystalline; PES, paclitaxel-eluting stent. 


\section{RESULTS}

\section{Tissue pharmacokinetics study}

Abdominal aortic tissue was harvested at different time points following either PCB dilation or TS implantation. The arterial tissue pharmacokinetic profile of all tested groups is shown in figure 3 . The overall vessel size was comparable among all groups and the paclitaxel tissue concentrations were normalised by total tissue weight (data not shown). Following PCB dilation, the $1 \mathrm{~h}$ total paclitaxel tissue concentrations were comparable with CC and AC. At $24 \mathrm{~h}, \mathrm{CC}$ sustained similar concentrations, whereas levels declined by $99 \%$ in $\mathrm{AC}$ group $(\mathrm{p}<0.01)$. In addition, at the same time point, both the CC (20-fold) and AC (5-fold) formulations displayed higher total paclitaxel tissue concentrations compared with the TS group $(\mathrm{p}<0.05)$. Within 7 days, there was a significant decline $(\mathrm{CC}=88.6 \%$ and $\mathrm{AC}=99.9 \%)$ in the total paclitaxel tissue concentrations compared with original $1 \mathrm{~h}$ concentration. In comparison, in the TS group there was an almost sixfold increase in the total paclitaxel tissue concentration at the same time point. In all groups, paclitaxel tissue levels remained detectable up to 28 days. The CC group resulted in tissue concentrations threefold higher than the TS group. Conversely, the $\mathrm{AC}$ group yielded paclitaxel tissue concentrations
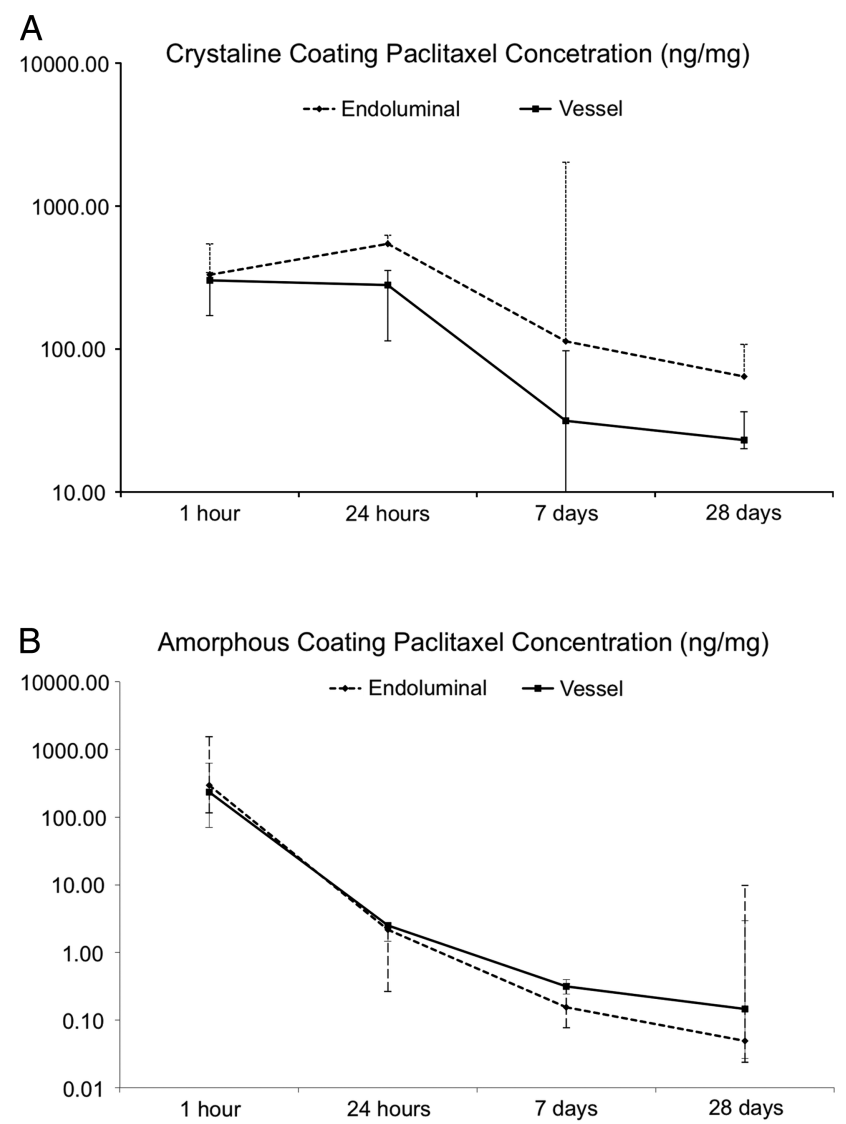

Figure 4 Compartmental analysis displaying the paclitaxel concentrations on the inner layer (endoluminal) and intra-arterial over time (crystalline (A) and amorphous (B) coatings). significantly lower and barely detectable when compared with the CC and the TS groups. Area under the pharmacokinetic curve was significantly higher in the CC group as compared with AC and PES (2422.7 vs 138.4 vs $102.45 ; \mathrm{p}<0.05)$. The calculated tissue half-life for paclitaxel delivered by the $\mathrm{CC}$ and the $\mathrm{AC}$ was 7.5 and 3.4 days, respectively.

\section{Paclitaxel vessel surface deposition analysis}

A compartmental vessel analysis (vessel surface vs arterial wall) was performed by scraping the vessel surface of the treated segments. In the CC group, paclitaxel concentrations were higher on the vessel surface when compared with arterial wall concentrations at $24 \mathrm{~h}, 7$ and 28 days (figure 4A). Furthermore, AUC for vessel surface paclitaxel concentration was significantly higher than the arterial wall level curve (AUC surface: 7118.8 vs intra-arterial: 1948.1, $\mathrm{p}=0.03)$. Conversely, in the $\mathrm{AC}$ group, the vessel surface and the arterial wall concentrations were similar at 1 and $24 \mathrm{~h}$ (figure $4 \mathrm{~B}$ ). After $24 \mathrm{~h}$, the vessel surface concentration declined further in comparison with the arterial wall levels (figure 4B). The AUCs for paclitaxel concentration were similar for both compartments in this group (AUC surface: 127.5 vs intra-arterial: $190.2 ; \mathrm{p}=0.2$ ). Additionally, at $1 \mathrm{~h}$, the total vessel surface paclitaxel presence appeared similar. However, at $24 \mathrm{~h}$ and at 7 days, the CC group retained a higher percentage of the initial paclitaxel uptake when compared with the AC group. A paclitaxel deposits distribution analysis is shown in figure 5 . This analysis demonstrated that approximately $27 \%$ of the total amount of drug delivered via balloon dilation remained on the surface of the vessel regardless of the coating type. At $24 \mathrm{~h}, 36.7 \%$ of the total paclitaxel recovered remained on the vessel surface in the CC group, compared with $13.1 \%$ in the AC group $(\mathrm{p}<0.01)$. In the $\mathrm{CC}$, the vessel surface deposits of paclitaxel remained stable up to 7 days, while these deposits further decreased in the AC group (38.4\% vs $11 \% ; \mathrm{p}=0.07$ ). At 28 days, the percentage of paclitaxel vessel surface deposits appeared to equalise between both groups. The calculated paclitaxel half-life for both compartments was similar in the AC group (2.5 days for vessel surface and intra-arterial). By contrast, in the CC group, the calculated vessel surface half-life was higher (11.5 days) compared with arterial wall (7.5 days).

\section{Tissue effects coronary study}

A summary of the imaging results is presented in table 1. At baseline, the reference vessel diameter and the stent:artery ratios were comparable between groups. The final minimal lumen diameter following stent implantation was also similar between groups. At 28-day follow-up, the CC group displayed larger minimal lumen diameters and lower percentage diameter stenosis compared with the AC and the bare balloon control groups. In the OCT in vivo evaluation, the CC group displayed larger lumen areas (increase $=63.8 \%$ vs $\mathrm{AC}$ and $45.9 \%$ vs plain balloon angioplasty (POBA); $\mathrm{p}=0.03$ ) and lower 
Figure 5 Paclitaxel endoluminal amount over time expressed as percentage of total vessel amount. AC, amorphous; CC, crystalline.

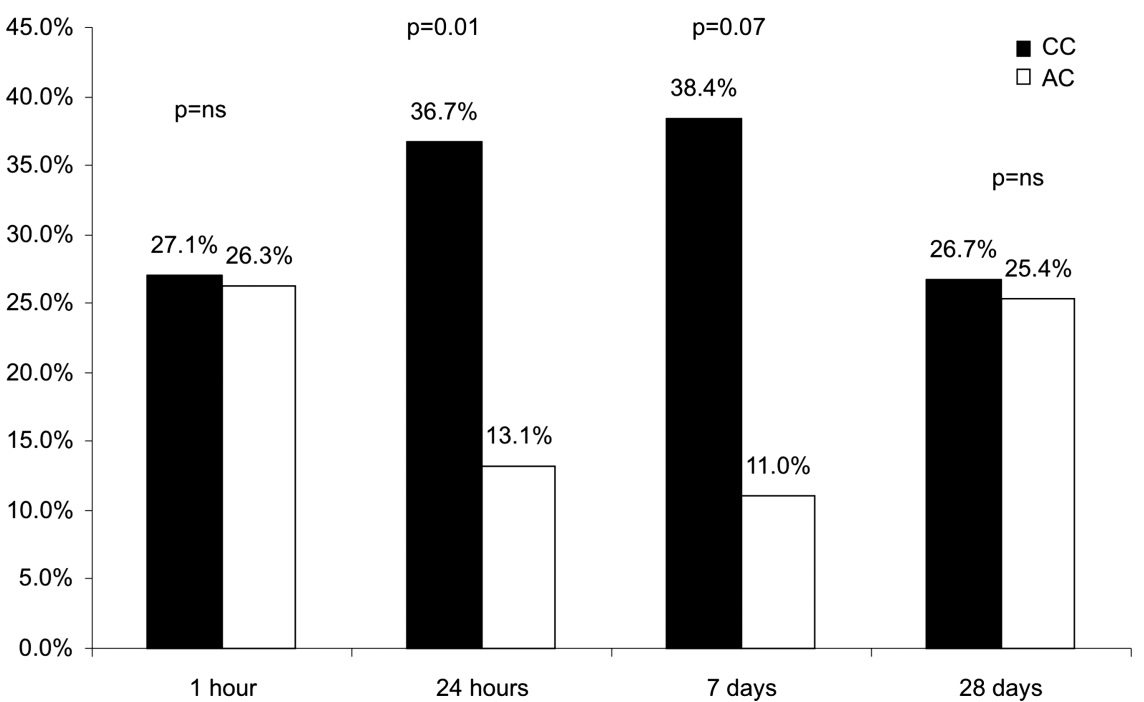

per cent area stenosis (decrease $=23.9 \%$ vs $\mathrm{AC}$ and $25 \%$ vs POBA; $\mathrm{p}=0.03$, table 2). In addition, OCT analysis showed that the distribution of neointima in the AC group was similar to that in the POBA control group (figure 6). Conversely, the CC group displayed a pattern of neointimal distribution that was heterogeneously distributed. A summary of the histological findings is shown in table 2 and representative stent cross sections in figure 7. Similar to the OCT findings, morphometric evaluation showed that the CC had $23.7 \%$ and $22.2 \%$ decrease in per cent area stenosis when compared with the AC and the POBA control group, respectively. The CC group displayed signs of drug effect such as slightly lower endothelialisation scores, less mature neointima and higher degrees of fibrin deposition in comparison to the AC and the POBA control groups.

\section{DISCUSSION}

The concept of developing a balloon-based drug delivery system to prevent arterial restenosis was based on the hypothesis that a durable effect on neointimal proliferation could be achieved following the single-time delivery of paclitaxel into the vessel wall. ${ }^{126} 15$ This early hypothesis contradicted previous experimental ${ }^{16}$ and clinical ${ }^{17}$ data suggesting that sustained tissue exposure to paclitaxel was necessary to effectively decrease restenosis. Owing to its unique delivery mechanism and proposed pharmacokinetic profile, the early promising clinical results of $\mathrm{PCB}$ technologies were originally challenged. ${ }^{18}$

Early experimental data confirmed that paclitaxel transfer into the vessel wall occurs rapidly following balloon inflation. ${ }^{6}{ }^{19}$ In addition, tissue pharmacokinetic

Table 1 Intravascular and angiographic imaging analyses at baseline and at 28-day follow-up following PCB delivery in porcine coronary arteries

Crystalline coating $(n=8) \quad$ Amorphous coating $(n=9) \quad$ POBA control $(n=9)$

\section{QVA}

Baseline

RVD (mm)

Stent to artery ratio

Balloon to artery ratio

Poststent MLD (mm)

OCT

28-day follow-up

$\operatorname{RVD}(\mathrm{mm})$

$\operatorname{MLD}(\mathrm{mm})$

Diameter stenosis (\%)

Lumen area $\left(\mathrm{mm}^{2}\right)$

Stent area $\left(\mathrm{mm}^{2}\right)$

Neointimal area $\left(\mathrm{mm}^{2}\right)$

Area stenosis (\%)

Neointimal thickness (mm)
$3.09 \pm 0.1$

$1.46 \pm 0.1$

$1.46 \pm 0.1$

$3.28 \pm 0.2$

$2.69 \pm 0.3$

$1.62 \pm 0.6$

$38.8 \pm 24$

$5.08 \pm 0.8^{\star}$

$10.65 \pm 0.4$

$5.57 \pm 0.7$

$53.1 \pm 14^{*}$

$0.63 \pm 0.1$
$3.00 \pm 0.1$

$1.35 \pm 0.1$

$1.41 \pm 0.1$

$3.08 \pm 0.3$
$3.05 \pm 0.1$

$1.48 \pm 0.1$

$1.46 \pm 0.1$

$3.13 \pm 0.2$

${ }^{*} \mathrm{p}<0.05$ vs Amorphous and POBA.

tp<0.05 vs Crystalline and POBA.

MLD, minimal lumen diameter; OCT, optical coherence tomography; PCB, paclitaxel-coated balloons; POBA, plain balloon angioplasty;

QVA, quantitative vessel analysis; RVD, reference vessel diameter. 
Table 2 Histomorphometric and vascular healing response analysis of stents according to the PCB formulation used at 28 days

\begin{tabular}{|c|c|c|c|c|}
\hline & Crystalline coating $(n=9)$ & Amorphous coating $(n=9)$ & POBA control $(n=8)$ & p Value \\
\hline \multicolumn{5}{|l|}{ Morphometric analysis } \\
\hline EEL area $\left(\mathrm{mm}^{2}\right)$ & $11.54 \pm 2.3$ & $9.53 \pm 1.2$ & $10.03 \pm 1.3$ & 0.054 \\
\hline IEL area $\left(\mathrm{mm}^{2}\right)^{\prime}$ & $9.29 \pm 1.5^{*}$ & $7.53 \pm 1.1$ & $7.85 \pm 1.4$ & 0.026 \\
\hline Lumen area $\left(\mathrm{mm}^{2}\right)$ & $4.25 \pm 1.9^{*}$ & $2.15 \pm 1.4$ & $2.47 \pm 2.0$ & 0.046 \\
\hline Medial area $\left(\mathrm{mm}^{2}\right)$ & $2.24 \pm 1.1$ & $2 \pm 0.3$ & $2.17 \pm 0.6$ & ns \\
\hline Neointimal thickness $(\mu \mathrm{m})$ & $588 \pm 232$ & $750 \pm 210$ & $759 \pm 283$ & ns \\
\hline Per cent area stenosis (\%) & $54.8 \pm 18^{*}$ & $71.8 \pm 15$ & $70.4 \pm 20$ & 0.05 \\
\hline \multicolumn{5}{|l|}{ Histological analysis } \\
\hline Injury score & $1.73 \pm 0.6$ & $1.88 \pm 0.7$ & $2.11 \pm 0.8$ & ns \\
\hline Peri-strut inflammation & $2.27 \pm 0.8$ & $2.32 \pm 0.7$ & $2.15 \pm 1.1$ & ns \\
\hline Adventitial inflammation & $1.44 \pm 0.7$ & $1.93 \pm 0.4$ & $1.79 \pm 1.1$ & ns \\
\hline Peri-strut granuloma & $2.52 \pm 0.9$ & $2.74 \pm 0.6$ & $2.42 \pm 1.1$ & ns \\
\hline Endothelial coverage & $2.63 \pm 0.5$ & $2.85 \pm 0.4$ & 3 & ns \\
\hline Fibrin deposits & $1.15 \pm 0.7^{*}$ & $0.15 \pm 0.3$ & 0 & $<0.01$ \\
\hline Neointima maturity & $2.41 \pm 0.5^{\star}$ & $2.96 \pm 0.1$ & 3 & $<0.01$ \\
\hline Neointima hypocellularity & $0.37 \pm 0.5$ & $0.11 \pm 0.2$ & 0 & ns \\
\hline Media hypocellularity & 0 & $0.04 \pm 0.1$ & 0 & ns \\
\hline
\end{tabular}

studies ${ }^{10}$ showed that short-term tissue levels of paclitaxel following balloon delivery were higher by several orders of magnitude compared with drug-eluting stents $^{20} 21$ and that therapeutic concentrations of paclitaxel were found beyond 28 days following initial drug delivery. ${ }^{5}$ Also, several publications demonstrated that the long-term vessel healing profile appears to be similar to commercially available drug eluting stent technologies. ${ }^{22}$ However, despite its documented clinical efficacy in selected clinical scenarios, a potential mechanism of action explaining long-term drug retention and sustained biological effect has not been proposed for PCB technologies. In these series of experimental studies, we propose a working hypothesis for the mechanism of action of PCB by comparing the pharmacokinetic

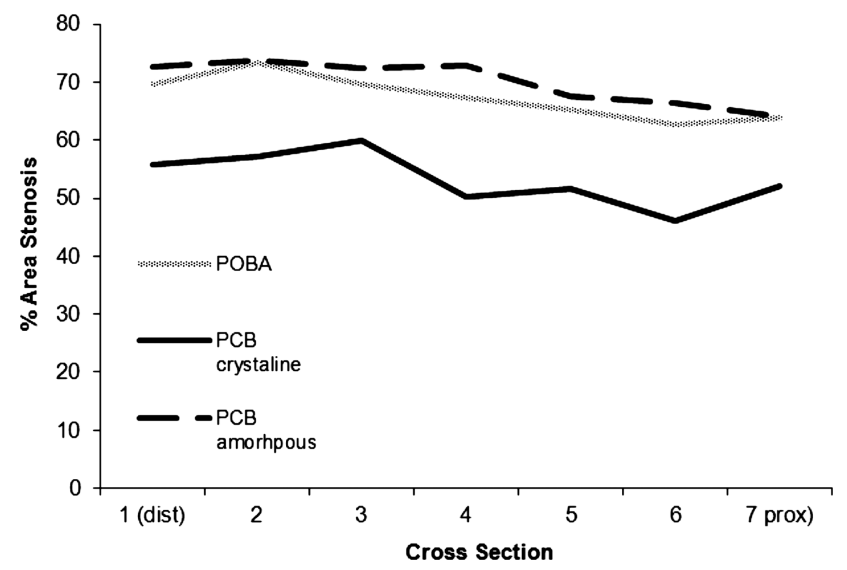

Figure 6 Neointimal distribution along the treated arterial segments by optical coherence tomography at 28 days in the tissue effects arm of the study. PCB, paclitaxel-coated balloon; POBA, plain balloon angioplasty. behaviour of two different coatings displaying opposite solubility profiles with a commercially available PES.

\section{Paclitaxel transfer and retention}

In the present study, paclitaxel tissue levels shown in the TS group followed a typical pattern of drug release from a polymer reservoir and the levels progressively increased from $24 \mathrm{~h}$ to 28 days (6-fold and 18-fold increase in tissue levels at 7 and 28 days, respectively). Data reported from a similar study in rabbit iliac arteries using $\mathrm{TS}^{23}$ demonstrated that paclitaxel tissue concentration increased from $0 \%$ at implant to approximately $5 \%$ at 30 days, and then decreased slowly through 180 days. Therefore, we chose 1, 7 and 28 days to model TS tissue paclitaxel increase to peak concentration. In contrast, paclitaxel transferred to the vessel wall via both PCB formulations was $\sim 20$ orders of magnitude higher compared with the early TS tissue levels (figure 3). At $24 \mathrm{~h}$, the tissue levels achieved by PCB using the $\mathrm{CC}$ remained stable. Conversely, in the AC group, there was a sharp decline in paclitaxel tissue levels achieving values comparable to the TS group. In the AC group, tissue paclitaxel levels continued to decline over time and fell below the TS curve at 28 days. Conversely, although CG tissue levels steadily continued to decline, the 28-day tissue levels achieved were comparable to the TS group. This finding may explain the lack of efficacy of some PCB technologies in early clinical trials. ${ }^{24}$ Our data also suggest that acute drug transfer and long-term tissue retention are independent of each other as both PCB formulations achieved comparable acute transfer levels but very different long-term tissue retention levels. In our study, the calculated tissue elimination half-life of total paclitaxel in treated arteries was several orders of magnitude higher 
A

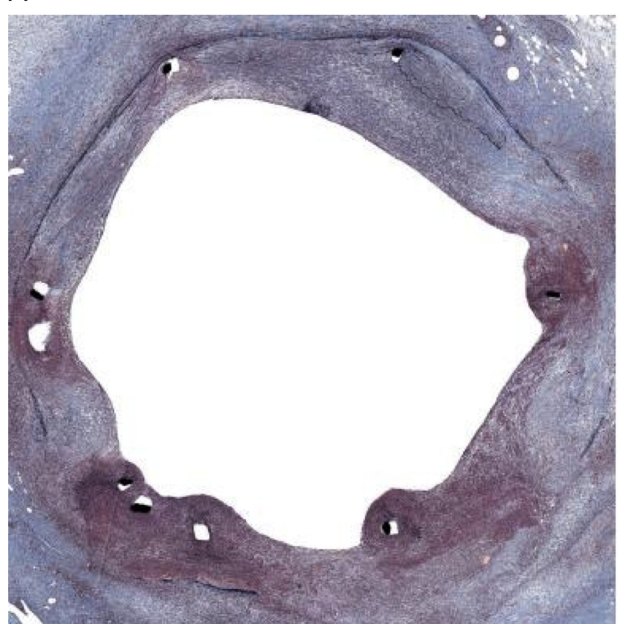

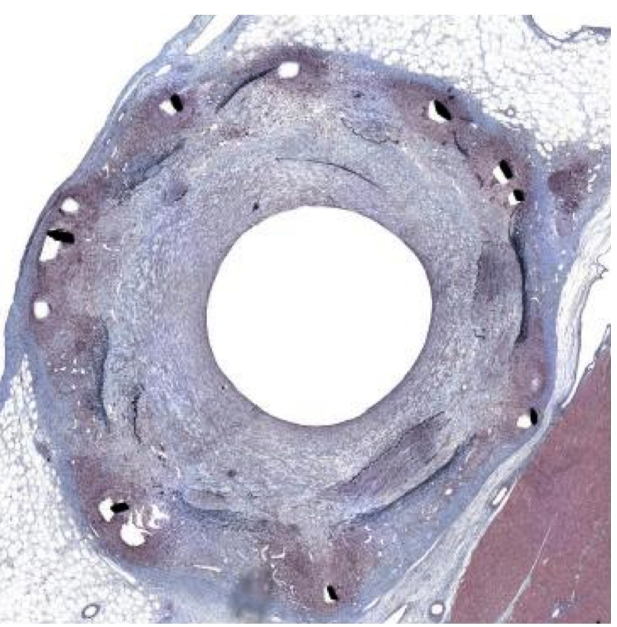

Figure 7 Representative histological pictures of $C C(A)$ and $A C(B)$ at 28 days in porcine coronary arteries following paclitaxel-coated balloon delivery.

(3.4 for $\mathrm{AC}$ and 7.5 for $\mathrm{CC}$ ) compared with previous experimental pharmacokinetic studies of paclitaxel delivered via balloon in which tissue elimination half-life is reported to be on the order of hours. ${ }^{12} 25-28$ This prolonged elimination half-life of paclitaxel suggests an additional mechanism for long-term retention through the development of compartments or deposits from which drug releases over time into the tissue.

\section{Vessel surface paclitaxel deposits and tissue retention}

In this study, we also demonstrated that following PCB dilation, a proportion of the paclitaxel is retained on the vessel surface and is not acutely dissolved into the tissue. In the CC group, sustained paclitaxel vessel surface levels were maintained, while in the AC group, the surface concentrations rapidly decreased over time. It seemed apparent that arterial wall levels of paclitaxel were driven by the sustained retention of drug on the surface of the vessel wall, thereby maintaining a positive concentration gradient from the vessel surface into the arterial wall. By $24 \mathrm{~h}$ the differences in paclitaxel vessel surface levels were striking between both PCB groups. However, by 7 days arterial tissue concentrations began to equalise the vessel surface levels providing an explanation about the lack of tissue toxic effects seen in experimental studies despite the apparent supratherapeutic tissue levels found immediately after PCB use. ${ }^{5} 611{ }^{29}$ At 30 days, the arterial tissue levels of paclitaxel seen in the CC group were comparable to the levels seen in the TS group and within the inhibitory concentration range of SMC proliferation. ${ }^{30}$ In addition, the concentration of paclitaxel found on the vessel surface in the CC group at the same time point was threefold higher than the arterial wall levels of the drug. These results seem to be consistent with previous reports showing that specific binding to intracellular proteins occurs primarily in the subintimal space and determines arterial transport properties and microtubule binding of paclitaxel. ${ }^{31} 32$

\section{Therapeutic window of PCB delivery}

Our data suggest that the observed tissue half-life of paclitaxel delivered by PCB technologies is rate limiting and relates to the slow dissolution of paclitaxel deposits from the vessel surface into arterial tissue in a time-dependent fashion. Once paclitaxel is transferred into the vessel wall, the mechanisms of intra-arterial transfer follow welldescribed patterns of pharmacokinetics. ${ }^{33}$ In any case, the resulting tissue levels of paclitaxel at 28 days are above the reported $\mathrm{IC}_{50}$ values for human SMC (1.4$2 \mathrm{ng} / \mathrm{g})^{30}$ and endothelial cell proliferation (1.7-6.8 $\mathrm{ng} /$ g) ${ }^{34}$ Thus, the proposed mechanism of action reconciles the apparent contradiction between the observed shortterm supratherapeutic tissue levels seen right after balloon delivery and the resulting vessel healing profiles seen at the experimental level. The 'total' paclitaxel levels measured in pharmacokinetic tissues are likely magnified due to the higher concentrations of nonsoluble paclitaxel deposits remaining on the surface. These deposits, important as a reservoir to maintain longterm tissue levels, appear to get washed off into the blood stream within the first 7 days following initial drug delivery. Then, the resulting levels of intra-arterial paclitaxel appear to be within the therapeutic range for inhibition of smooth muscle proliferation and below cytotoxic concentrations, which is consistent with experimental and clinical data, both for peripheral and coronary territory, on safety and efficacy reported to date. ${ }^{1-415}$

\section{Biological response to paclitaxel transfer}

The differences in paclitaxel tissue retention found in both PCB groups resulted in significant differences in the degrees of neointimal formation between both groups. In general, the pattern of drug release and retention displayed by the CC group resulted in higher total vessel luminal areas, heterogeneity of neointimal formation, SMC loss and fibrin deposits. In addition, in vivo OCT analysis demonstrated a more heterogeneous 
pattern of neointimal formation in the CC group. In conclusion, in these series of studies, we demonstrated that the type of paclitaxel coating impacts the degree of drug transfer and retention. Long-term paclitaxel retention appears to depend on surface deposits that serve as delivery mechanism occurring over time.

\section{Study limitations}

The main limitation of this study derives from its experimental nature and the analytical methods used. First, the normal rabbit aorta lacks the biological components seen in atherosclerotic vascular disease. Therefore, the pharmacokinetic results found in our studies may not correlate with the levels potentially found in humans. It is well known that there is a significant amount of variability seen in pharmacokinetic studies using animal models. In addition, since efficiency of removal of insoluble drug by the surface wipe technique is not perfect, the obtained values may introduce analytical errors and may not reflect accurate values for the percentage of total drug in the surface deposition study. However, all studies were performed following identical protocols and procedures introducing the same systematic errors, if any. Finally, the stent study used a different animal species (porcine) and slightly higher balloon to artery ratio than commonly performed in the experimental setting. Although this approach was used in order to increase the biological response to drug delivery and healing, it is known that this method may introduce significant variability of the resulting data. Importantly, since each PCB uses different manufacturing

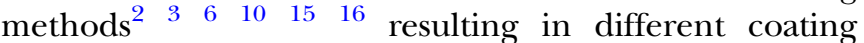
characteristics, the results of the present study may not be directly representative of all PCB technologies.

Contributors JFG contributed to the concept, planning, conducting and manuscript drafting, and he is the guarantor. MS contributed to the concept and planning. PPB was involved in planning, data analysis and interpretation, statistics and manuscript drafting. AT was involved in conducting, data and pathological analysis. DL was involved in planning. GLK was involved in planning and study management. MBL was involved in planning and scientific supervision. WG and MRJ were involved in data interpretation and manuscript revision. RSS was involved in scientific supervision, concept and planning.

Funding MEDRAD Interventional (Indianola, PA) provided financial support and materials for this study.

Competing interests At the time of experiment, MS and DL were full time employees of Medrad Interventional.

Ethics approval The Institutional Animal Care and Use Committee approved the study.

Provenance and peer review Not commissioned; externally peer reviewed.

Data sharing statement No additional data are available.

Open Access This is an Open Access article distributed in accordance with the Creative Commons Attribution Non Commercial (CC BY-NC 4.0) license, which permits others to distribute, remix, adapt, build upon this work noncommercially, and license their derivative works on different terms, provided the original work is properly cited and the use is non-commercial. See: http:// creativecommons.org/licenses/by-nc/4.0/

\section{REFERENCES}

1. Scheller B, Hehrlein C, Bocksch W, et al. Treatment of coronary in-stent restenosis with a paclitaxel-coated balloon catheter. $N$ Engl J Med 2006;355:2113-24.

2. Tepe G, Zeller T, Albrecht T, et al. Local delivery of paclitaxel to inhibit restenosis during angioplasty of the leg. $N$ Engl $\mathrm{J}$ Med 2008;358:689-99.

3. Werk M, Langner S, Reinkensmeier B, et al. Inhibition of restenosis in femoropopliteal arteries: paclitaxel-coated versus uncoated balloon: femoral paclitaxel randomized pilot trial. Circulation 2008;118:1358-65.

4. Latib A, Colombo A, Castriota $F$, et al. A randomized multicenter study comparing a paclitaxel drug-eluting balloon with a paclitaxel-eluting stent in small coronary vessels: the BELLO (Balloon Elution and Late Loss Optimization) study. J Am Coll Cardiol 2012;60:2473-80.

5. Speck U, Cremers B, Kelsch B, et al. Do pharmacokinetics explain persistent restenosis inhibition by a single dose of paclitaxel? Circ Cardiovasc Interv 2012;5:392-400.

6. Scheller B, Speck U, Abramjuk C, et al. Paclitaxel balloon coating, a novel method for prevention and therapy of restenosis. Circulation 2004;110:810-14.

7. Joner M, Finn AV, Farb A, et al. Pathology of drug-eluting stents in humans: delayed healing and late thrombotic risk. J Am Coll Cardiol 2006;48:193-202.

8. Ong AT, McFadden EP, Regar E, et al. Late angiographic stent thrombosis (LAST) events with drug-eluting stents. J Am Coll Cardiol 2005;45:2088-92.

9. Stone GW, Moses JW, Ellis SG, et al. Safety and efficacy of sirolimus- and paclitaxel-eluting coronary stents. N Engl J Med 2007;356:998-1008.

10. Posa A, Hemetsberger R, Petnehazy O, et al. Attainment of local drug delivery with paclitaxel-eluting balloon in porcine coronary arteries. Coron Artery Dis 2008;19:243-7.

11. Radke PW, Joner M, Joost $A$, et al. Vascular effects of paclitaxel following drug-eluting balloon angioplasty in a porcine coronary model: the importance of excipients. Eurolntervention 2011;7:730-7.

12. Yeh TK, Lu Z, Wientjes MG, et al. Formulating paclitaxel in nanoparticles alters its disposition. Pharm Res 2005;22:867-74.

13. Institute of Laboratory Animal Resources NRC. Principles of care of laboratory animals. NIH Publication, 1996:85-23.

14. Hanley JA, McNeil BJ. The meaning and use of the area under a receiver operating characteristic (ROC) curve. Radiology 1982;143:29-36.

15. Unverdorben M, Vallbracht C, Cremers B, et al. Paclitaxel-coated balloon catheter versus paclitaxel-coated stent for the treatment of coronary in-stent restenosis. Circulation 2009;119:2986-94.

16. Ferns GA, Avades TY. The mechanisms of coronary restenosis: insights from experimental models. Int J Exp Pathol 2000;81:63-88.

17. Serruys PW, Sianos G, Abizaid A, et al. The effect of variable dose and release kinetics on neointimal hyperplasia using a novel paclitaxel-eluting stent platform: the Paclitaxel In-Stent Controlled Elution Study (PISCES). J Am Coll Cardiol 2005;46:253-60.

18. Henry TD, Schwartz RS, Hirsch AT. "POBA Plus": will the balloon regain its luster? Circulation 2008;118:1309-11.

19. Scheller B, Speck U, Schmitt A, et al. Addition of paclitaxel to contrast media prevents restenosis after coronary stent implantation. J Am Coll Cardiol 2003;42:1415-20.

20. Stampfl U, Radeleff B, Sommer C, et al. Paclitaxel-induced arterial wall toxicity and inflammation: part 2-long-term tissue response in a minipig model. J Vasc Interv Radiol 2009;20:1608-16.

21. Radeleff B, Lopez-Benitez R, Stampfl U, et al. Paclitaxel-induced arterial wall toxicity and inflammation: tissue uptake in various dose densities in a minipig model. J Vasc Interv Radiol 2010;21:1262-70.

22. Cremers B, Milewski K, Clever YP, et al. Long-term effects on vascular healing of bare metal stents delivered via paclitaxel-coated balloons in the porcine model of restenosis. Catheter Cardiovas Interv 2012;80:603-10.

23. Miller KM. A drug-eluting stent case study: TAXUS Express2-from development to approval. New England Chapter Parental Drug Association Workshop. 2004.

24. Cortese B, Micheli A, Picchi A, et al. Paclitaxel-coated balloon versus drug-eluting stent during $\mathrm{PCl}$ of small coronary vessels, a prospective randomised clinical trial. The PICCOLETO study. Heart 2010;96:1291-6.

25. Innocenti F, Danesi R, Di Paolo A, et al. Plasma and tissue disposition of paclitaxel (taxol) after intraperitoneal administration in mice. Drug Metab Dispos 1995;23:713-17.

26. Eiseman JL, Eddington ND, Leslie J, et al. Plasma pharmacokinetics and tissue distribution of paclitaxel in CD2F1 mice. Cancer Chemother Pharmacol 1994;34:465-71. 
27. Fetterly GJ, Straubinger RM. Pharmacokinetics of paclitaxelcontaining liposomes in rats. AAPS PharmSci 2003;5:E32.

28. Gustafson DL, Long ME, Bradshaw EL, et al. P450 induction alters paclitaxel pharmacokinetics and tissue distribution with multiple dosing. Cancer Chemother Pharmacol 2005;56:248-54.

29. Herdeg C, Oberhoff M, Baumbach A, et al. Local paclitaxel delivery for the prevention of restenosis: biological effects and efficacy in vivo. J Am Coll Cardiol 2000;35:1969-76.

30. Axel DI, Kunert W, Goggelmann C, et al. Paclitaxel inhibits arteria smooth muscle cell proliferation and migration in vitro and in vivo using local drug delivery. Circulation 1997;96:636-45.
31. Levin AD, Vukmirovic N, Hwang CW, et al. Specific binding to intracellular proteins determines arterial transport properties for rapamycin and paclitaxel. Proc Natl Acad Sci USA 2004;101:9463-7.

32. Creel CJ, Lovich MA, Edelman ER. Arterial paclitaxel distribution and deposition. Circ Res 2000;86:879-84.

33. Balakrishnan B, Dooley JF, Kopia G, et al. Intravascular drug release kinetics dictate arterial drug deposition, retention, and distribution. J Control Release 2007;123:100-8.

34. Pourroy B, Honore S, Pasquier E, et al. Antiangiogenic concentrations of vinflunine increase the interphase microtubule dynamics and decrease the motility of endothelial cells. Cancer Res 2006;66:3256-63. 